\title{
The thermal boundary resistance at semiconductor interfaces: A critical appraisal of the Onsager vs. Kapitza formalisms
}

\author{
Riccardo Rurali \\ Institut de Ciència de Materials de Barcelona (ICMAB-CSIC) Campus de Bellaterra, \\ 08193 Bellaterra, Barcelona, Spain. \\ Xavier Cartoixà \\ Departament d'Enginyeria Electrònica, \\ Universitat Autònoma de Barcelona, \\ 08193 Bellaterra, Barcelona, Spain. \\ Dick Bedeaux and Signe Kjelstrup \\ PoreLab, Department of Chemistry, \\ Norwegian University of Science and Technology, NO-7491 Trondheim, Norway. \\ Luciano Colombo* \\ Dipartimento di Fisica, Università di Cagliari, \\ Cittadella Universitaria, I-09042 Monserrato (Ca), Italy.
}

\begin{abstract}
We critically readdress the definition of thermal boundary resistance at an interface between two semiconductors. By means of atomistic simulations we provide evidence that the widely used Kapitza formalism predicts thermal boundary resistance values in good agreement with the more rigorous Onsager non-equilibrium thermodynamics picture. The latter is, however, better suited to provide physical insight on interface thermal rectification phenomena. We identify the factor ruling over the actual temperature profile across the interface and the source of interface thermal rectification. To this end we perform non-equilibrium molecular dynamics computational experiments on a Si-Ge system with a graded compositional interface of varying thickness, considering thermal bias of different sign.
\end{abstract}

*Electronic address: luciano.colombo@unica.it 


\section{INTRODUCTION}

Understanding heat transport across an interface between two semiconductors is both a subtle thermodynamic problem and a topic central to nanotechnology [1-4]. When a temperature gradient is applied across a junction between two materials with unlike thermal transport properties, a sudden temperature drop $\Delta T$ can occur, highlighting a thermal boundary resistance (TBR), usually referred to as the Kapitza resistance [5]. The need to understand and predict its value is a key feature in nanotechnology, since TBR affects any thermal exchange at the nanoscale and, therefore, rules over the actual thermal budget of any nanodevice.

The Kapitza resistance is defined as $R_{\text {Kapitza }}^{\mathrm{TBR}}=-\Delta T / J$ (dimension $\mathrm{K} \mathrm{m} \mathrm{m}^{2} / \mathrm{W}$ ), where $\Delta T$ is evaluated across the thickness of the interface (the right minus the left hand side value; see also Fig. 1), and the positive heat transport $J$ takes place in the direction of the temperature drop. The negative difference in temperature is in most cases regarded as the driving force for heat transport across interfaces or heterogeneous systems. And in most cases, this gives sufficient accuracy and consistency. The thermodynamic driving force according to Onsager for heterogeneous systems is, however, the difference in the inverse temperature across the interface [6]. The linear laws that arise in the two cases are obviously different. One may therefore wonder if one of the forms should be preferred to the other. In other words, are there theoretical or practical reasons to prefer one to the other? The present paper shall attempt to answer these questions. In particular, we want to examine the role of the interface temperature, which can be well defined for interfaces if we follow the recipe of Gibbs.

We have earlier found that the TBR is a function of the interface temperature [7], which depends on the thermal bias across the heterojunction that separates the two materials: if their bulk thermal resistances are different, the interface temperature changes upon reversal of the thermal bias. This situation has been addressed as thermal rectification [8, 9]. It is due to an excess contribution of the interface over the two constituent materials. The purpose of the present work is to add to the fundamental understanding of TBR, including thermal rectification phenomena.

Among the methods developed so far to calculate TBR, we have chosen molecular dynamics (MD) simulations for a twofold reason, namely: neither (i) the direct use of the phonon 
language is needed, nor (ii) do we need to guess the scattering mechanisms of heat carriers at the interface; in addition, (iii) the method is not biased versus any of the thermodynamic descriptions, as it involves only the solution of Newton's laws. While the first feature is beneficial in treating systems that are lacking translational invariance, the second and third enable the derivation of a very general picture, not relying on any pre-guessed microscopic mechanism for energy dissipation.

In the determination of the Kapitza value of the TBR, it has normally been assumed that a steady-state condition has been established, corresponding to a constant thermal current crossing the interface. We shall work under such conditions also here. This has the advantage that we can express the total interface resistance as the sum of resistances that are connected in series.

Customarily, the TBR of a given semiconductor heterojunction is calculated, according to the Kapitza formulation, by a separate evaluation of the $\Delta T$ and $J$, once that a given thermal bias has been imposed to the system and a steady-state regime has been established [10-12]. Both quantities are straightforwardly addressed by MD simulations. The same data shall here also be evaluated within the formalism of non-equilibrium thermodynamics [6], using the numerical implementation already successfully exploited in Ref. [7]. We focus on the show-case of a graded (i.e. smooth) Si/Ge interface, namely a prototypical example of semiconductor heterojunction of paramount important in nanotechnology.

The paper is organized as follows: in Sec. II we define the system of interest, set up the simulation protocol, and give the relevant equations; in Sec. III we present and discuss our results; conclusions and a critical comparison between Kaptiza and Onsager formulations are eventually drawn in the final Sec. IV.

\section{THEORETICAL AND COMPUTATIONAL METHODS}

\section{A. System}

The system is schematically represented in Fig. 1. There is a graded interface between Si (gray dots) and Ge (orange dots) taken to be along the [100] crystallographic direction (hereafter referred to as the $z$ direction), see figure top. The switch from pure-Si to pure-Ge leads is obtained by a linear variation of the stoichiometry within an embedded SiGe layer of 
total thickness $\Delta z$. This geometry is convenient for the present theoretical investigation. It means that there is no excess of any component ( $\mathrm{Si}$ or $\mathrm{Ge}$ ) relative to the equimolar surface of the other component in the interface. It also corresponds to an experimental situation of interest for applications $[13,14]$.

A pseudomorphic structure is assumed with lattice constant equal to $5.55 \AA$. Periodic boundary conditions are imposed along the directions perpendicular to the temperature gradient. The simulation cell is characterized by a cross sectional area of $30.85 \mathrm{~nm}^{2}$ and a total $z$-length of $63 \mathrm{~nm}$. The constant area $A$, but not the length $\Delta z$, is used in the calculation of resistivities.

It is common to define the interface as the region where the compositions differ from the value characterizing both elemental materials. The Gibbs construction [6] is described below. We shall use Gibbs definition of the interface, which allows us to assess its autonomous thermodynamical nature [7].

It is expected that, if we apply a temperature gradient along $z$ and wait until a steadystate regime has been reached, a temperature profile $T(z)$ is established, as shown in Fig. 1, bottom. It was demonstrated in Ref. [7] that there is no reason for such a temperature profile to be symmetrically shaped within the interface region nor centered at its nominal position $\Delta z / 2$. Both features are emphasized in Fig. 1, bottom for illustration purposes (the resulting picture, however, is not in scale).

\section{B. Simulation protocol}

We have studied heat transport by non-equilibrium molecular dynamics (NEMD) in the above Si/Ge system using the bond-order Tersoff [15] potential, as implemented in the LAMMPS code [16]. Equations of motions have been integrated by the velocity-Verlet algorith with a timestep as short as $0.7 \mathrm{fs}$. Each stationary condition has been established at first by connecting both ends of the computational cell with two Nosé-Hoover thermostats at temperatures $T_{\text {hot }}$ and $T_{\text {cold }}$, respectively. The system was then aged for $5.25 \mathrm{~ns}$; all stationary quantities have been eventually averaged over the last 3 ns. The length of the simulation was chosen so as to provide a reasonably converged estimate of the TBR, following the criteria established in Ref. [17].

In order to quantitatively identify the interface and its thickness $\Delta z$ we have carefully 
reproduced the procedure leading to the Gibbs construction for a Si/Ge interface described in Ref. [7]. In particular, we have performed a structural relaxation and tracked how the average first-neighbour distance varied upon $z$ : variations of more than two standard deviations from the Si and Ge bulk values have been used to estimate, respectively, the left and right interface boundary.

Once the interface was determined, we calculated its temperature $T_{\text {int }}$ from the average kinetic energy (in stationary conditions) $\left\langle E_{k}^{\text {int }}\right\rangle$ of the atoms therein as:

$$
T_{\text {int }}=\frac{2\left\langle E_{k}^{\text {int }}\right\rangle}{3 k_{B}}
$$

where $k_{B}$ is the Boltzmann constant. The temperatures $T_{\text {left }}$ and $T_{\text {right }}$ have been defined by separately fitting $T(z)$ in the $\mathrm{Si}$ and Ge regions (i.e. sufficiently far away from the interface and the thermostats) and linearly extrapolating these bulk-like values to the interface boundaries. All relevant temperatures in our problem (namely: $T_{\text {left }}, T_{\text {right }}$ and $T_{\text {int }}$ ) are illustrated in Fig.1.

The heat flux integrated over the (constant) cross-section was computed from the power injected (extracted) by the hot (cold) thermostat once the steady-state is reached. After a suitable transient period, these two quantities were equal within numerical accuracy, and did not further vary with time: both features were taken as an additional proof that the stationary regime was indeed reached.

\section{Formulating the TBR}

The Kapitza resistance was defined above, see also [1, 5], as (dimension $\mathrm{K} \mathrm{m}^{2} \mathrm{~W}^{-1}$ )

$$
R_{\text {Kapitza }}^{\mathrm{TBR}}=\frac{T_{\text {left }}-T_{\text {right }}}{J}
$$

With the formalism of nonequilibrium thermodynamics of heterogeneous systems [6, 18] we define the Onsager coefficient

$$
R_{\text {Onsager }}^{\mathrm{TBR}}=\frac{1}{J}\left(\frac{1}{T_{\text {right }}}-\frac{1}{T_{\text {left }}}\right)
$$

Notice that this coefficient has different dimensions, namely $\mathrm{m}^{2} W^{-1} \mathrm{~K}^{-1}$, from the conventional Kapitza resistance. Neverthelss, for the sake of simplicity, we indistinctly refer to it 
as resistance or Onsager resistance throughout the rest of the paper. By comparing the two expressions above, we find that they are related by

$$
R_{\text {Kapitza }}^{\mathrm{TBR}}=T_{\text {right }} T_{\text {left }} R_{\text {Onsager }}^{\mathrm{TBR}}
$$

A common feature is that both formulations rely on linear response: Kapitza is simply related to Fourier's law, Onsager because it assumes a linear relationship between thermodynamical forces and the related fluxes. Furthermore, as for their determination, they similarly require the evaluation of the steady-state heat flux $J$ and the temperatures $T_{\text {left }}$ and $T_{\text {right }}$ at the interface boundaries.

For the Onsager coefficient, an additional feature applies. Given that the interface has been determined according to Gibbs, we can assume that the interface is an autonomous thermodynamic system and thus its properties are univocally determined by local variables, such as the interface temperature, $T_{\text {int }}$. Furthermore, we know from Onsager that the resistivity is a function of the state variables, but not of the driving forces. In the present case there is only one state variable, the temperature $T_{\text {int }}$, since there is no excess masses in the interface. We know therefore that $R_{\text {Onsager }}^{\mathrm{TBR}}$ is a unique function of $T_{\text {int }}$.

The existence of an interface temperature allows us splitting the Onsager resistance into contributions from each side of the interface $\left(r^{\text {left }}\right.$ and $r^{\text {right }}$, respectively)

$$
\left(\frac{1}{T_{\text {int }}}-\frac{1}{T_{\text {left }}}\right)=r^{\text {left }} J \quad\left(\frac{1}{T_{\text {right }}}-\frac{1}{T_{\text {int }}}\right)=r^{\text {right }} J
$$

The contributions add to the total thermal boundary resistivity $R_{\text {Onsager }}^{\mathrm{TBR}}$.

$$
R_{\text {Onsager }}^{\mathrm{TBR}}=r^{\mathrm{left}}+r^{\mathrm{right}}
$$

The three resistivities in this equation are all functions of $T_{\mathrm{int}}$.

According to Eq.4, $R_{\mathrm{Kapitza}}^{\mathrm{TBR}}$ does not have a unique dependence on any interface temperature. It depends on the driving force through the product $T_{\text {right }} T_{\text {left }}$. This is a conceptually different situation from the Onsager coefficient. The Kapitza coefficient has no similar theoretical basis. In order to explore its dependence on both temperatures, we write (cf. Fig.1)

$$
T_{\text {left }}=T_{\text {int }}+\Delta T_{\text {left }} \quad \text { and } \quad T_{\text {right }}=T_{\text {int }}-\Delta T_{\text {right }}
$$

where the net temperate drop across the interface is $\Delta T=T_{\text {left }}-T_{\text {right }}=\Delta T_{\text {left }}+\Delta T_{\text {right }}$. Accordingly we can state that

$$
T_{\text {right }} T_{\text {left }}=T_{\text {int }}^{2}+T_{\text {int }}\left(\Delta T_{\text {left }}-\Delta T_{\text {right }}\right)-\Delta T_{\text {left }} \Delta T_{\text {right }}
$$


and therefore, we find convenient to define the dimensionless variable

$$
\frac{T_{\text {int }}^{2}}{T_{\text {left }} T_{\text {right }}}=\left(1+\frac{\Delta T_{\text {left }}-\Delta T_{\text {right }}}{T_{\text {int }}}-\frac{\Delta T_{\text {left }} \Delta T_{\text {right }}}{T_{\text {int }}^{2}}\right)^{-1}
$$

which accounts for the difference between the Kapitza definition of the TBR and $R_{\text {Onsager }}^{\mathrm{TBR}} T_{\text {int }}^{2}$, where we take this modified version of the Onsager resistance to make a direct comparison with $R_{\mathrm{Kapitza}}^{\mathrm{TBR}}$ possible. This factor can be used to find that this difference is $\mathcal{O}\left(\frac{\Delta T}{T_{\text {int }}}\right)$ in the interface temperature offset. A rationale for the rectification behavior of the $\mathrm{Si} / \mathrm{Ge}$ graded interface will emerge from the comparative investigation of the two contributions

\section{RESULTS AND DISCUSSION}

We have presented two alternative definitions of TBR (Eqs.2 and 3). For the very same system and overall thermal conditions, $J$ is linear in the temperature difference in the Kapitza formulation (Eq.2), while in the Onsager formulation it is linear in the difference of the corresponding inverse temperatures. By a linear law, in non-equilibrium thermodynamics, it is meant that the dependence on the driving force of the transport coefficients is linear. It might well depend on state variables, like the temperature or composition. For large differences, the relation may therefore also become non-linear.

We have investigated both formulations in the specific case of an interface with $\Delta z=$ $15 \mathrm{~nm}$ when $T_{\text {left }}>T_{\text {right }}$ as well as when $T_{\text {right }}>T_{\text {left }}$. The comparison was done keeping the interface temperature $T_{\text {int }}$ constant $[6,7]$. While this is conceptually clean, in practice it required a carefully balanced thermostatting procedure implemented far away from the interface: the actual $T_{\text {hot }}$ and $T_{\text {cold }}$ temperatures were tuned in such a way that the corresponding $T_{\text {int }}$ value was basically unchanged, nevertheless corresponding to different offsets between $T_{\text {right }}$ and $T_{\text {left }}$ or, equivalently, between $1 / T_{\text {right }}$ and $1 / T_{\text {left }}$.

In Fig. 2 we report the variation of the steady-state heat flux as function of a difference of temperatures (panel a) or difference of inverse temperatures (panel b) in the positive bias condition $T_{\text {left }}>T_{\text {right }}$ defined with respect to Fig. 1. Similarly, in Fig. 3 we report the trends calculated in the negative bias condition $T_{\text {left }}<T_{\text {right }}$. Symbols represent the output of the MD simulations and the full (panels a) or dashed (panels b) lines in both figures correspond to a linear fit of the Kapitza and Onsager [Eq. (3)] equations, respectively. 
Similar computations were repeated for a negative bias, with a much smaller interface temperature, cf. Fig. 4, where the $J$ trend vs. difference of temperatures (panel a) or difference of inverse temperatures (panel b) is shown for $T_{\text {int }}=98 \mathrm{~K}$.

In all cases, it is clear that neither of the schemes can be said to be much better than the other when it comes to linearity in the fitted data. This is to be expected for temperatures where $\left(T_{\text {int }}\right)^{2} \approx T_{\text {left }} T_{\text {right }}$, like in Fig. 2 and 3. But also the case of low temperatures, with $T_{\text {int }}=98 \mathrm{~K}$ give a similar picture. We conclude that the agreement between data and linear fit is rather good in both the Kapitza and the Onsager formulations. Deviations are, in fact, somewhat larger in the latter case.

It remains to discuss whether the given functional dependence on $T_{\text {int }}$ of the Onsager coefficient has any special advantage.

Although the phenomenological Kapitza theory summarized in Eq. (2) seems to be more accurate for prediction of a robust value of TBR, a comparison with the Onsager formulation (Eq.4) makes clear that the Kapitza coefficient depends on the driving force, which is easy to control. On the other hand, the Onsager coefficient is a property of the interface only. This makes it more suitable for tabulation and als more suited to understand the physics of the transport phenomena. We have previously seen that it can help explain interface rectification phenomena.

In the present case, the two contributions to $T_{\text {int }}^{2} / T_{\text {left }} T_{\text {right }}$ appearing in Eq. (8)are reported in Fig. 5 for both forward (black) and reverse (red) thermal bias. The main qualitative difference shown here is that the sign of the $\left(\Delta T_{\text {left }}-\Delta T_{\text {right }}\right) / T_{\text {int }}$ contribution changes by switching the thermal bias, while the $-\left(\Delta T_{\text {left }} \Delta T_{\text {right }} / T_{\text {int }}^{2}\right)$ contribution is always negative. While this is expected from the very definition of such terms, the physical consequence is interesting: the $T_{\text {int }}^{2} / T_{\text {left }} T_{\text {right }}$ term is very close to 1 in forward bias because of a nearly perfect cancellation of the two opposite contributions appearing in Eq. (9). On the other hand, the same correction grows almost monotonically (for $\Delta z>2.5 \mathrm{~nm}$ ) in negative bias providing a size-able amendment of $\sim 15 \%$ for the larger $\Delta z=15 \mathrm{~nm}$ interface thickness.

\section{CONCLUSIONS}

By treating a model $\mathrm{Si} / \mathrm{Ge}$ solid-solid junction prepared in a stationary thermal bias condition, we have shown that its thermodynamics is fully exploited by the Onsager picture 
for non-equilibrium transport coefficients. We furthermore reported a simulation protocol suited to compute the interface temperature.

We eventually provided a thermodynamical description of interface thermal resistance and rectification features.

While the Onsager prediction is consistent with the more simple picture provided by Kapitza as for of the thermal boundary resistance, it is the only theoretical framework able to offer a full thermodynamical explanation of the predicted non negligible thermal rectification properties of a graded $\mathrm{Si} / \mathrm{Ge}$ interface. Present findings are in full agreement with a previous investigation of ours, where rectification was attributed to the fact that the thermal conductivity of a graded SiGe system is a non-separable function of both temperature and position [19].

These results improve our fundamental understanding of heat transport phenomena across solid interfaces and, therefore, could be beneficial in many front-end nanotechnologies where either thermal fluxes reflect the most fundamental mechanism of energy exchange or excess of heat represents the key limiting factor affecting nanodevice performances.

\section{Acknowledgments}

We acknowledge financial support by the Ministerio de Economía y Competitividad (MINECO) under grants no. FIS2012-37549-C05-02, MAT2013-40581-P, TEC2015-67462C2-1-R (MINECO/FEDER) and FEDER-MAT2017-90024-P, the Generalitat de Catalunya under grants no. 2014 SGR 301 and 2017 SGR 1506, and the Spanish MINECO through the Severo Ochoa Centres of Excellence Program under Grants SEV-2013-0295 and SEV2015-0496. SK and DB are grateful to the Research Council of Norway through its Centres of Excellence funding scheme, project number 262644, PoreLab.

[1] G. L. Pollack, Rev. Mod. Phys. 41, 48 (1969).

[2] E. T. Swartz and R. O. Pohl, Rev. Mod. Phys. 61, 605 (1989).

[3] D. G. Cahill, W. K. Ford, K. E. Goodson, G. D. Mahan, A. Majumdar, H. J. Maris, R. Merlin, and S. R. Phillpot, J. Appl. Phys. 93, 793 (2003). 
[4] D. G. Cahill, P. V. Brown, G. Chen, D. R. Clarke, S. Fan, K. E. Goodson, P. Keblisnki, W. P. King, G. D. Mahan, A. Majumdar, et al., Appl. Phys. Reviews 1, 011305 (2014).

[5] P. L. Kapitza, J. Phys.(USSR) 4, 181 (1941).

[6] S. Kjelstrup and D. Bedeaux, in Non-Equilibrium Thermodynamics of Heterogeneous Systems (World Scientific, Singapore, 2008), vol. 16.

[7] R. Rurali, L. Colombo, X. Cartoixà, W. Øivind, T. T. Trinh, D. Bedeaux, and S. Kjelstrup, Phys. Chem. Chem. Phys. 18, 13741 (2016).

[8] D. C., J. Heat Transf. 131, 061301 (2009).

[9] N. Roberts and D. Walker, Int. J. Thermal Sci. 50, 648 (2011).

[10] V. Samvedi and V. Tomar, Nanotechnology 20, 365701 (2009).

[11] E. S. Landry and A. J. H. McGaughey, Phys. Rev. B 80, 165304 (2009).

[12] E. S. Landry and A. J. H. McGaughey, J. Appl. Phys. 107, 013521 (2010).

[13] L. Llin, A. Samarelli, S. Cecchi, T. Etzelstorfer, E. M. Gubler, D. Chrastina, G. Isella, J. Stangl, J. Weaver, P. Dobson, et al., Appl. Phys. Lett. 103, 143507 (2013).

[14] K. Hahn, S. Cecchi, and L. Colombo, Appl. Phys. Lett. 108, 203102 (2016).

[15] J. Tersoff, Phys. Rev. B 39, 5566 (1989).

[16] S. Plimpton, J. Comp. Phys. 117, 1 (1995), URL http://lammps.sandia.gov/.

[17] R. Rurali, X. Cartoixà, and L. Colombo, Phys. Rev. B 90, 041408 (2014).

[18] R. Dettori, C. Melis, X. Cartoixà, R. Rurali, and L. Colombo, Adv. Phys. X 1, 246 (2016).

[19] R. Dettori, C. Melis, R. Rurali, and L. Colombo, J. Appl. Phys. 119, 215102 (2016). 


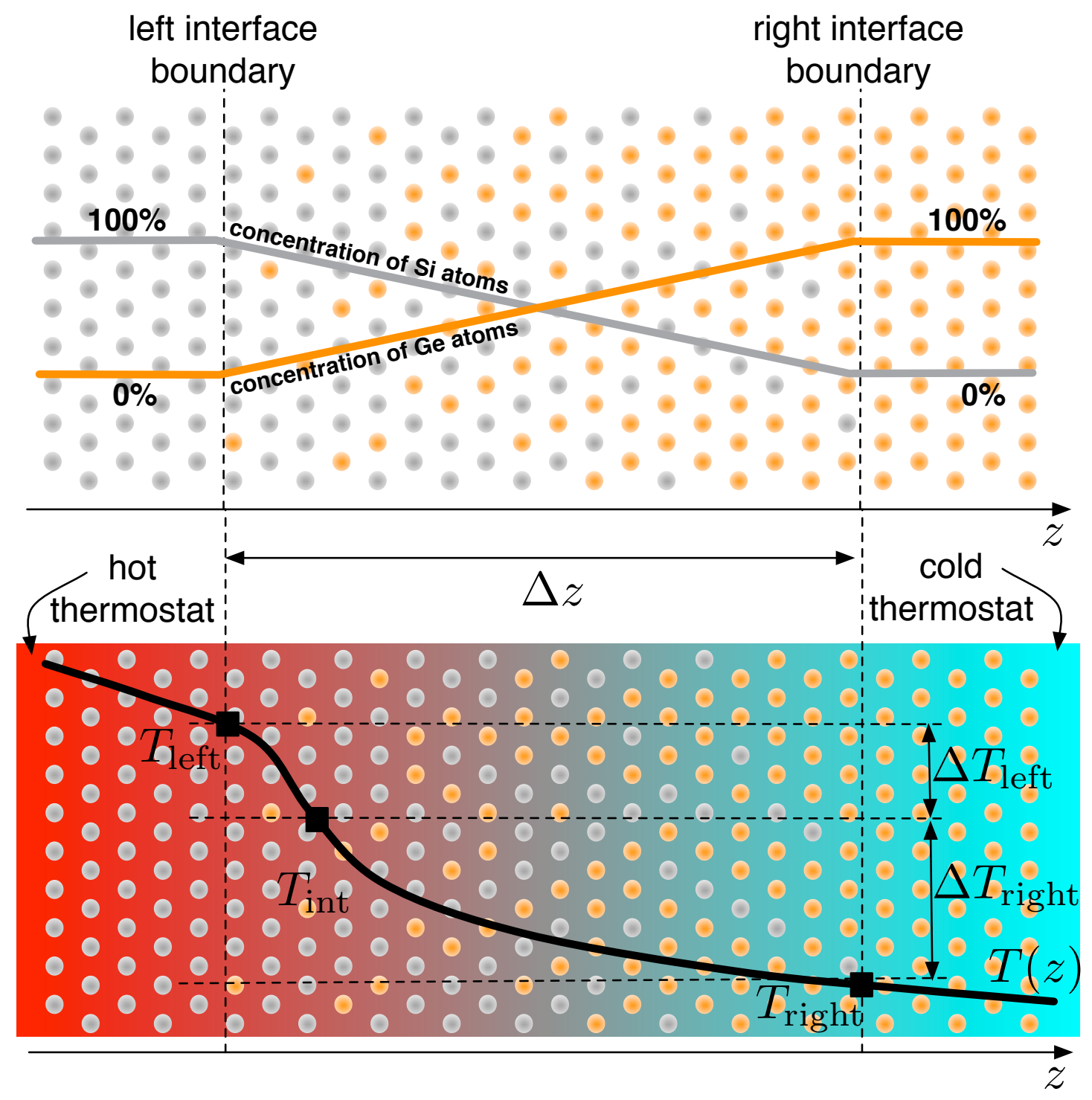

Figure 1: (color online)Top: An interface between bulk Si (left, Si atoms correspond to grey dots) and a bulk Ge (right, Ge atoms correspond to orange dots) is obtained by a linear variation of the stoichiometry within an embedded SiGe layer, for which a pseudomorphic (with a lattice constant of $5.55 \AA$ ) and defect-free crystallography is assumed. Bottom: the stationary temperature profile $T(z)$ (full black line) established by an applied thermal bias. Temperatures $T_{\text {left }}, T_{\text {right }}$, and $T_{\text {int }}$ are defined as the temperature at the left and right boundary of the interface region, respectively, and as the average kinetic temperature within the interface volume. 

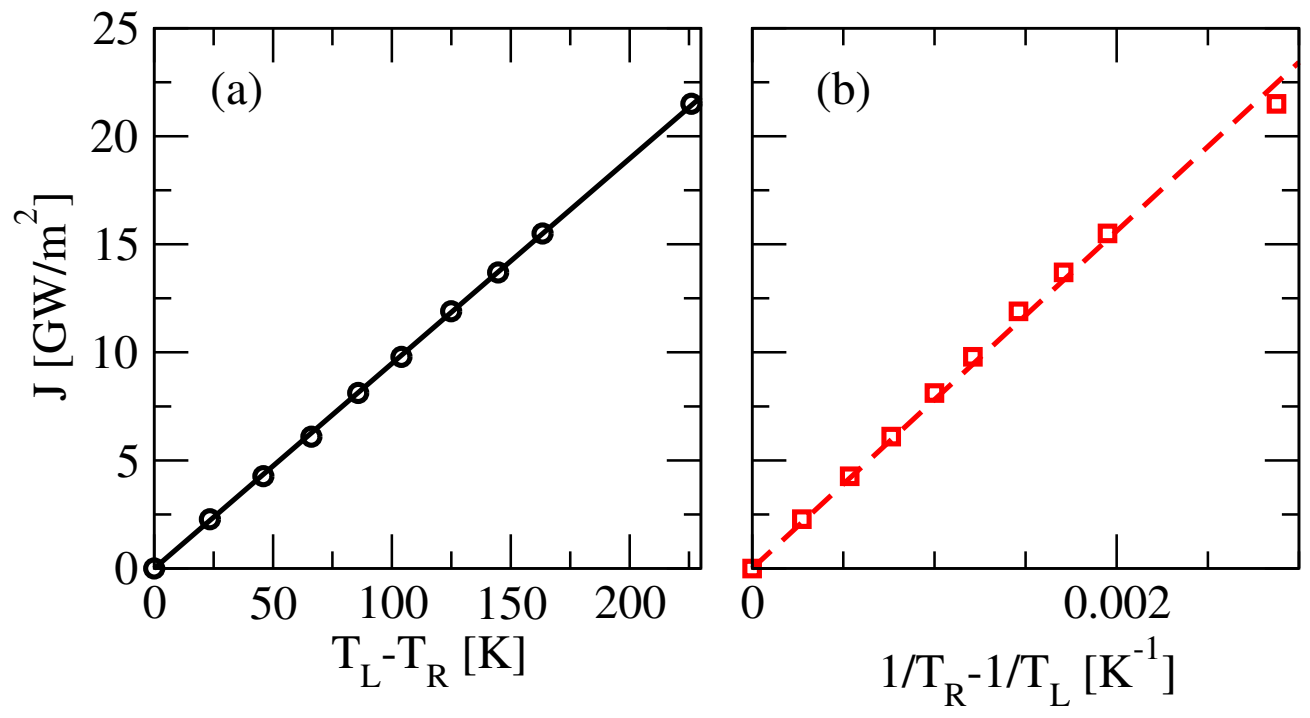

Figure 2: (color online)Variation of the steady-state heat flux $J$ as function of a difference of temperatures (panel a) or difference of inverse temperatures (panel b) in the positive bias condition $T_{\text {left }}>T_{\text {right }}$ defined with respect to Fig. 1 . Symbols represent the output of the MD simulations, while the full (panel a) or dashed (panel b) line corresponds to a linear fit to the Kapitza or Onsager equation, respectively. All data correspond to an interface temperature contained in the range $291.4 \mathrm{~K}<T_{\text {int }}<293.0 \mathrm{~K}$. 

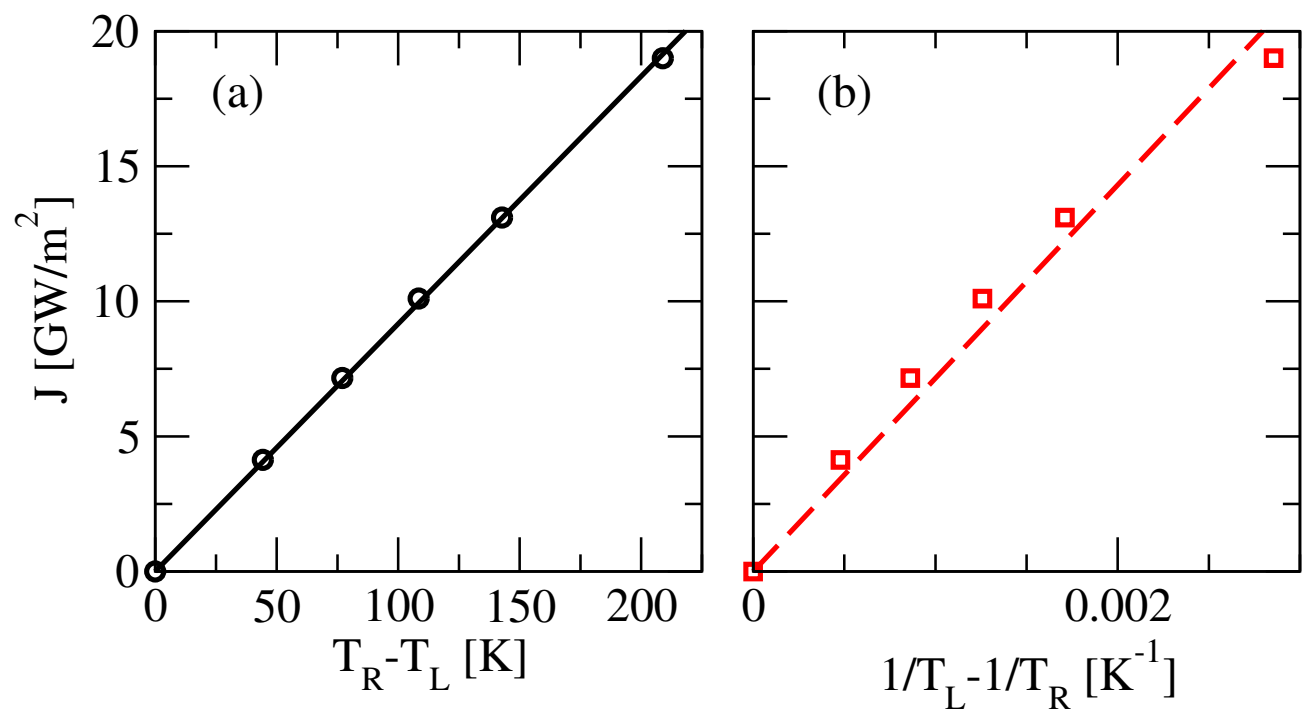

Figure 3: (color online)Variation of the steady-state heat flux $J$ as function of a difference of temperatures (panel a) or difference of inverse temperatures (panel b) in the negative bias condition $T_{\text {left }}<T_{\text {right }}$ defined with respect to Fig. 1 . Symbols represent the output of the MD simulations, while the full (panel a) or dashed (panel b) line corresponds to a linear fit to the Kapitza or Onsager equation, respectively. All data correspond to an interface temperature contained in the range $306.6 \mathrm{~K}<T_{\text {int }}<308.1 \mathrm{~K}$. 


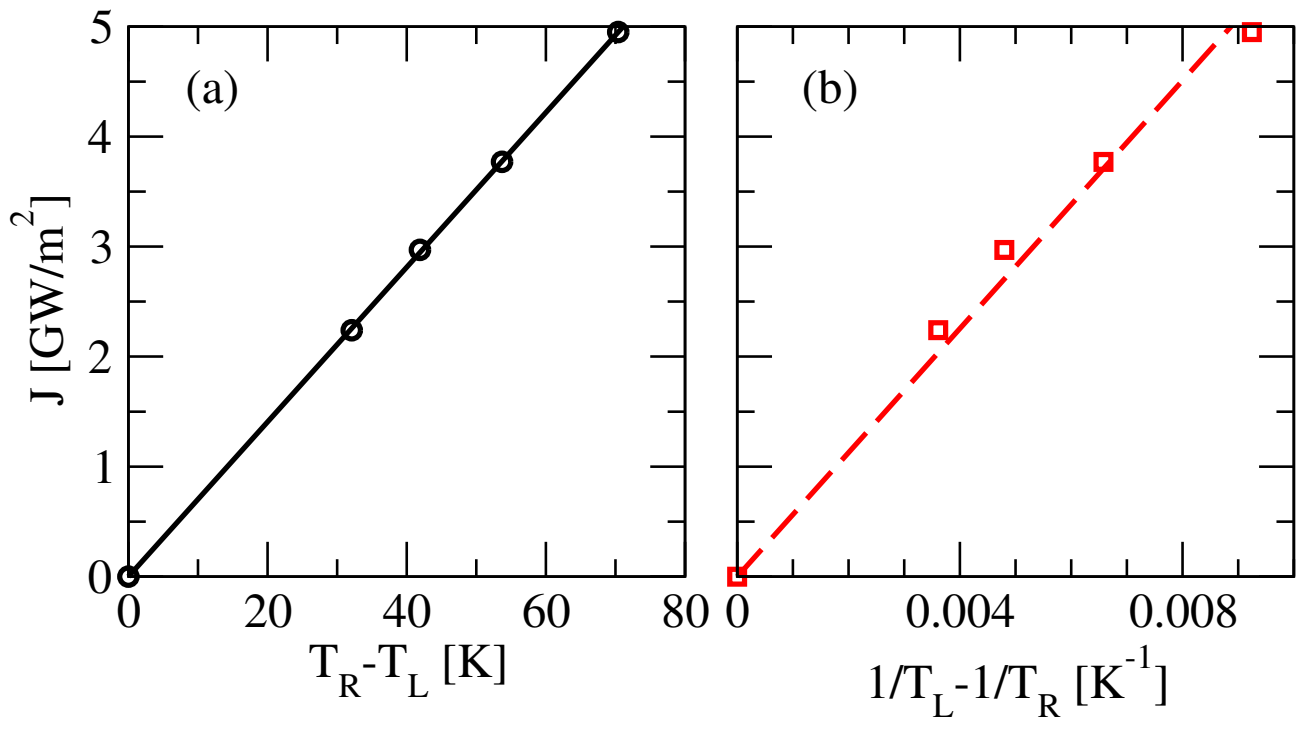

Figure 4: (color online) Variation of the steady-state heat flux $J$ as function of a difference of temperatures (panel a) or difference of inverse temperatures (panel b) in the negative bias condition $T_{\text {left }}<T_{\text {right }}$ defined with respect to Fig.1. Symbols represent the output of the MD simulations, while the full (panel a) or dashed (panel b) line corresponds to a linear fit to the Kapitza or Onsager equation, respectively. All data correspond to an interface temperature contained in the range $97.1 \mathrm{~K}<T_{\text {int }}<98.1 \mathrm{~K}$. 

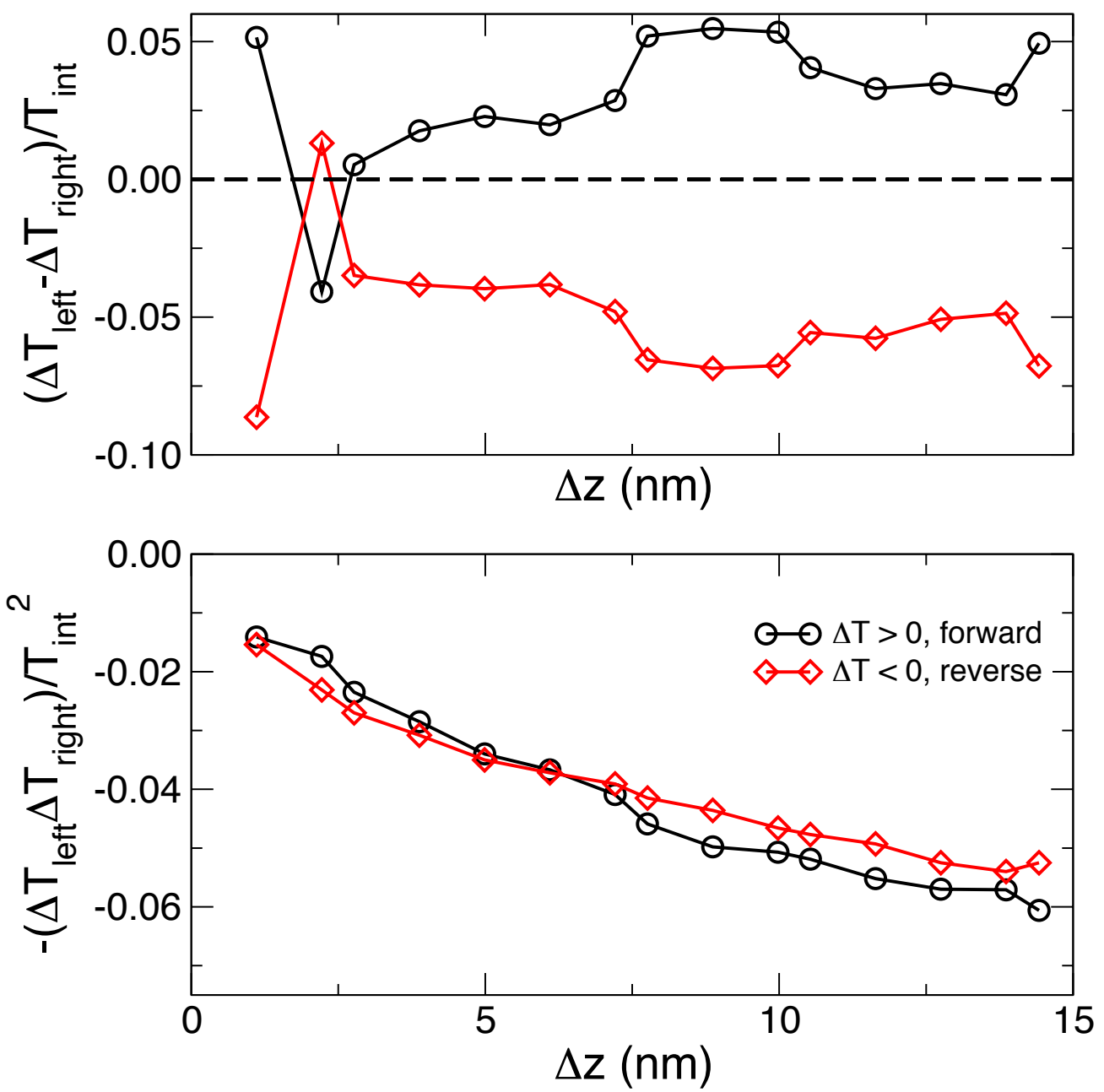

Figure 5: The two contributions to $T_{\text {int }}^{2} / T_{\text {left }} T_{\text {right }}$ appearing in eq.( $(7)$. 